\title{
Dor lombar, flexibilidade muscular e relação com o nível de atividade física de trabalhadores rurais
}

\author{
Low back pain, muscle flexibility and relationship with the level of \\ physical activity of rural workers
}

Marcia Regina da Silva1, Fátima Ferretti², Junir Antonio Lutinski³

RESUMO O objetivo foi investigar a dor lombar, flexibilidade muscular e relação com o Nível de Atividade Física (NAF) de trabalhadores rurais. Pesquisa transversal com 184 trabalhadores rurais, 44,24 $( \pm 10,83)$ anos. Avaliou-se o NAF pelo Questionário Internacional de Atividade Física (Ipaq); dor e disfunção lombar pela Escala Visual Analógica da dor (EVA) e Índice de Incapacidade Oswestry (IIO); avaliação da Flexibilidade Toracolombar (FBW) e de isquiotibiais (Ângulo Poplíteo - AP - e Teste de Elevação da Perna Retificada - TEPR). Os dados foram analisados pelos testes Kruskal Wallis, seguido pelo U de Mann-Whitney e correlação de Spearman. Houve correlação negativa entre a EVA com a FBW, AP e TEPR. A variação da dor pela EVA foi alta, e indivíduos sedentários possuem menor flexibilidade de isquiotibiais.

PALAVRAS-CHAVE Dor lombar. Amplitude de movimento articular. Esforço físico. População rural. Fisioterapia.

ABSTRACT The aim was to investigate low back pain, muscle flexibility and relationship with the Level of Physical Activity (LPA) of rural workers. It is a cross-sectional survey with 184 rural workers, 44.24 ( \pm 10.83$)$ years. We evaluated the LPA by Ipaq; pain and lumbar dysfunction by Visual Analog Scale (VAS) and Oswestry disability index (ODI); evaluation of thoracolumbar

1 Universidade Comunitária da Região de Chapecó (Unochapecó), Graduação em Fisioterapia - Chapecó (SC), Brasil.

marciaf@unochapeco.edu.br

2 Universidade Comunitária da Região de Chapecó (Unochapecó), PósGraduação em Ciências da Saúde - Chapecó (SC), Brasil.

ferrettifisio@yahoo.com.br

3 Universidade Comunitária da Região de Chapecó (Unochapecó), Pós-

Graduação em Ciências da Saúde - Chapecó (SC) Brasil.

junir@unochapeco.edu.br flexibility (TF) and hamstrings (PA and SLR). The data were analysed by Kruskal Wallis test followed by Mann-Whitney test and Spearman correlation. There was a negative correlation between the VAS with the TF, PA and SLR. The variation of pain by VAS was high and sedentary individuals have less flexibility of hamstring.

KEYWORDS Low back pain. Range of motion, articular. Physical exertion. Rural population. Physical therapy specialty. 


\section{Introdução}

A saúde do trabalhador tem-se constituído em um tema central de pesquisas no campo da saúde pública, investigando as condições de trabalho que podem predispor ao adoecimento. A pesquisa realizada por Moreira et al. (2015) buscou analisar a saúde de trabalhadores da atividade agrícola no Brasil, identificando a percepção de saúde e as morbidades referidas no banco da Pesquisa Nacional por Amostra de Domicílios (PNAD), e evidenciou que a ocupação agrícola diminuiu a chance dessa população referir sua saúde como boa e aumentou a chance de referir doenças na coluna vertebral, enfermidade que geralmente traz a dor como sintoma associado.

As causas mais comuns de adoecimentos nos trabalhadores rurais estão relacionadas com as excessivas demandas físicas do trabalho, sendo que as Doenças Osteomusculares (Dort) são as que mais afetam os trabalhadores, ocasionando dor lombar (ALVES; GUIMARÃES, 2012). A elevação e transporte de cargas pesadas, flexão e extensão prolongada e repetida da coluna e movimentos repetitivos estão entre os fatores de risco para o desenvolvimento de lesões e quadros álgicos (FATHALLAH, 2010). Entre os processos de dor, destacam-se as lombalgias, em função da alta incidência, pois se estima que entre $60 \%$ e $80 \%$ dos indivíduos, em geral, sofrem de sintoma de dor lombar em algum momento da vida, sendo mais comum entre os 25 e 60 anos de idade (HAMILL; KNUTZEN, 2012). Estudo na região sul do Brasil identificou prevalência de $63,1 \%$ de dor nas costas, sendo a região lombar a mais referida (40\%) (FERREIRA ET AL., 2011).

Estudos apontam que a adoção de uma vida ativa se constitui em fator de proteção para uma vida saudável, sendo que quanto mais ativo um indivíduo for, menor será o número de enfermidades (VIDMAR; POTULSKI; SACHETTI, 2011; MATSUDO; MATSUDO; BARROS NETO, 2011). Esses autores entendem que a atividade física é um fator de proteção funcional para a vida humana, e por isso necessária, em todas as faixas etárias. Reforçando essa questão, para manter-se ativo, há que se manter boa flexibilidade, componente essencial da aptidão física que, associada a níveis adequados de força, melhora a eficiência do movimento e reduz a incidência de distensão muscular, aspecto fundamental para evitar quadros álgicos (HAMILL; KNUTZEN, 2012). Tanto a força quanto a flexibilidade muscular são frequentemente relacionadas com a dor lombar, especialmente quando há retração de isquiotibiais, banda iliotibial; fraqueza da musculatura abdominal e eretores espinhais (HAMILL; KNUTZEN, 2012), o que pode predispor a uma maior incidência de quadros dolorosos.

O trabalho no ambiente rural envolve atividades físicas como caminhadas frequentes, transportes de materiais e produtos, levantamento de peso e o contato direto com agentes físicos, químicos e biológicos de diferentes naturezas. Assim, o desenvolvimento de agravos à saúde, como dores lombares e a perda da flexibilidade, podem estar associadas à sobrecarga de atividades relacionadas com o trabalho e com a falta de atividades preventivas ou compensatórias. Diante do contexto em que o trabalhador rural se encontra exposto, o presente estudo buscou investigar a dor lombar, flexibilidade de isquiotibiais e toracolombar e sua relação com o Nível de Atividade Física (NAF) de trabalhadores rurais de um município catarinense, bem como correlacionar a dor lombar com a flexibilidade de isquiotibiais e toracolombar e dor lombar com o tempo de atividade agrícola no período e fora do período de safra.

\section{Material e métodos}

Pesquisa de caráter quantitativo-observacional, com o delineamento de um estudo de corte transversal, desenvolvida em um município do Extremo Oeste catarinense que possuí aproximadamente 4.400 habitantes, 
com característica demográfica predominantemente rural. A amostra do estudo foi composta por trabalhadores rurais de ambos os sexos, na faixa etária de 20 a 59 anos, que se encontravam em plena atividade. Para cálculo amostral, considerou-se a população na faixa etária da amostra pretendida, registrada no Censo Demográfico de 2010, totalizando 1.316 indivíduos (IBGE, 2016). Com base na população, estimou-se a amostra em 298 indivíduos, adotando como nível de confiança $95 \%$ e erro amostral de $5 \%$.

Foram excluídos do estudo os trabalhadores que estavam fora da faixa etária estipulada, que não trabalhavam no ramo agropecuário, que não foram encontrados no domicílio em nenhuma de três tentativas consecutivas ou que não concordaram em participar do estudo. De acordo com os critérios de inclusão e exclusão, a amostra foi composta por 174 trabalhadores rurais.

Para a coleta de dados, foi utilizado um questionário com perguntas sobre o perfil e caracterização ocupacional dos trabalhadores rurais. Em seguida, foram aplicados outros dois instrumentos para verificação da dor lombar: Índice de incapacidade Oswestry (IIO) e Escala Visual Analógica (EVA) da dor, além da verificação do NAF (Ipaq - Questionário Internacional de Atividade Física).

O IIO é composto de 10 seções que descrevem dor ou limitações resultantes da lombalgia. Cada seção possui seis itens que descrevem um grau crescente de severidade, sendo zero o indicativo de pequena ou nenhuma dor e/ou limitação funcional e o escore de cinco, que indica dor e/ou limitação extrema. A pontuação total foi obtida somando-se o número de pontos de cada seção, sendo que a pontuação máxima corresponde ao escore de 50. O percentual de dor e/ ou limitação foi obtido multiplicando-se a pontuação atingida por 2, sendo este o maior grau de limitação percebido pelo paciente. Por meio dessa pontuação, o grau de disfunção do paciente foi classificado em: mínimo (de zero a 20 pontos), moderado (de 21 a
40 pontos) e severo (acima de 41 pontos) (MASSELI; LOPES; SERILLO, 2003).

A EVA consiste de uma escala graduada com linha horizontal de 10 centímetros de comprimento, sendo que na extremidade à esquerda possui um número correspondente a zero, com a expressão 'sem dor', e à direita, o número 10, com a expressão 'dor insuportável'. A dor pode ser classificada como leve quando a escala for de 1 a 3; moderada, de 4 a 6 e forte, de 7 a 9. A dor zero representa a ausência de dor e que os indivíduos não têm problemas para desempenhar suas atividades; na dor leve, é possível desempenhar as atividades, embora tenha dor; a dor moderada atrapalha parcial ou totalmente as atividades e há prejuízo no desempenho e a dor forte impede a realização das atividades (CARVALHO; KOVACS, 2006).

O questionário Ipaq foi utilizado para quantificar o NAF dos trabalhadores. Ele é composto por 5 seções, sendo: atividade física no trabalho; atividade física como meio de transporte; atividade física em casa (trabalho, tarefas domésticas e cuidar da família); atividade física de recreação, esporte, exercício e de lazer e tempo gasto sentado (MATSUDO ET AL., 2001). O questionário é classificado em: sedentário, irregularmente ativo, ativo ou muito ativo, considerando a pontuação obtida pela soma da quantidade de dias e minutos ou horas das atividades realizadas com base nos critérios: frequência, intensidade e duração das atividades (ZANCHETTA ET AL., 2010).

Posteriormente, foi realizado o teste de Flexibilidade Toracolombar (FBW) e de isquiotibiais. A flexibilidade toracolombar foi obtida por meio da flexão anterior de tronco sentado, utilizando o Banco de Wells Sanny ${ }^{\circledR}$. A partir da posição sentada sobre os ísquios com os joelhos em completa extensão, tornozelos em dorsiflexão com as plantas dos pés apoiadas no banco, os sujeitos flexionavam o tronco até o limite da sua Amplitude de Movimento (ADM), deslizando os dedos sobre a régua do banco de Wells, mantendo os 
joelhos estendidos. A distância total alcançada representou o valor total da flexibilidade adquirida, considerando a média de três tentativas (GAYA; SILVA, 2009; BERTOLLA ET AL., 2007).

A flexibilidade de isquiotibiais foi obtida pelos testes de comprimento muscular utilizando goniômetro $\mathrm{Carci}^{\circledR}$, por meio da medida do Ângulo Poplíteo (AP) e Teste de Elevação da Perna Retificada (TEPR). A medida do AP foi obtida com o sujeito posicionado em decúbito dorsal horizontal, o quadril e joelho do membro inferior a ser testado eram mantidos fletidos a $90^{\circ}$, o membro inferior contralateral em extensão completa sobre o solo ou mesa de exame, não sendo permitida a flexão de joelho e quadril. A seguir, o joelho do membro a ser testado era estendido ativamente, com o tornozelo e pé em abandono, até o ponto máximo em que o sujeito não apresentasse compensações com a coluna ou membro contralateral (AFFONSO FILHO; NAVARRO, 2002). A medida foi realizada três vezes, obtendo-se a média final entre elas. A medida era considerada normal quando estivesse entre $165^{\circ}$ e $180^{\circ}$ de ADM.

O TEPR foi realizado com o sujeito posicionado em decúbito dorsal, com membros inferiores estendidos, coluna lombar e sacro contra a mesa/maca. Mantendo a posição inicial, o participante realizou uma flexão de quadril com o joelho em extensão (elevação da perna) ao seu máximo, sem haver compensações. Um ângulo de aproximadamente $80^{\circ}$ entre a mesa e o quadril fletido foi considerado amplitude normal de comprimento dos isquiotibiais (KENDALL; MCCREARY; PROVANCE, 2007).

A análise de dados foi realizada pelo programa SPSS versão 20.0. Inicialmente, obteve-se estatística descritiva das variáveis estudadas. Após, foi realizado o teste de Shapiro-Wilk para verificação da normalidade das variáveis de idade, dor, flexibilidade e número de horas de atividades agrícolas. Os valores medianos da dor e flexibilidade de isquiotibiais e toracolombar foram comparados nos três grupos, formados a partir da classificação do Ipaq, no total de trabalhadores, por meio do teste de Kruskal Wallis seguido de comparações múltiplas com o teste U de Mann-Whitney. Os mesmos testes foram utilizados separadamente para comparar os grupos compostos por homens e mulheres. A correlação entre a dor pela escala EVA e a flexibilidade de isquiotibiais e toracolombar, e dor com o número de horas relatadas pelos agricultores no período de safra e fora do período de safra, foi realizada pelo teste de correlação de Spearman. Foi considerado como valor de significância estatística: $\mathrm{p}<0,05$.

A pesquisa foi apreciada e aprovada pelo Comitê de Ética em Pesquisa com Seres Humanos da Instituição de Ensino, sob protocolo $n^{\circ} 156 / 14$, com base na Resolução $n^{\circ}$ 466/12 do Conselho Nacional de Saúde.

\section{Resultados}

Dos 174 trabalhadores rurais avaliados, $44,3 \%(n=77)$ eram homens, e $55,7 \%(n=97)$ eram mulheres; 93,7\% $(n=163)$ de descendência italiana, 90,8\% $(\mathrm{n}=158)$ casados. A média de anos de estudo foi de 7,22 $( \pm 3,11)$ anos, variando entre 1 e 16 anos, e a média de tempo em que moravam no ambiente rural foi de 29,39 $( \pm 14,27)$ anos.

Quanto às tarefas realizadas na propriedade, destacou-se: lidar com animais (47,7\%), citado tanto pelos homens (41,6\%) quanto pelas mulheres $(52,6 \%)$, seguido de cuidar da horta (20,1\%) e múltiplas tarefas (14,9\%).

A média de tempo que os trabalhadores relataram utilizar para a realização de atividades agrícolas no período de safra foi de $11,63( \pm 2,5)$ horas, e fora do período da safra foi $6,75( \pm 1,84)$ horas.

Quanto à dor lombar, quase a totalidade dos agricultores $(98,3 \%)$ relataram ter algum sintoma de dor lombar, sendo que $100 \%$ das mulheres e $96,1 \%$ dos homens acusaram a presença de dor. Quanto ao grau de disfunção de Oswestry, 78,2\% (n=136) foram classificados com disfunção leve, e apenas $6,9 \%$ 
com disfunção severa $(\mathrm{n}=12)$, sendo que os resultados são similares entre homens e mulheres. A média da intensidade da dor classificada pela EVA foi $5,89( \pm 2,49)$, sendo mais alta nas mulheres $(6,14 \pm 2,45)$ do que nos homens $(5,56 \pm 2,51)$.

A tabela 1 demonstra as características da ocorrência da dor conforme a EVA e sua classificação nos trabalhadores em geral, separados por gênero.

Tabela 1. Ocorrência da dor conforme classificação pela Escala Visual Analógica (EVA)

\begin{tabular}{lrrr}
\hline Variável & $\begin{array}{c}\text { Total }(\mathbf{n}=\mathbf{1 7 4}) \\
\mathbf{n}(\%)\end{array}$ & $\begin{array}{c}\text { Masculino }(\mathbf{n}=\mathbf{7 7}) \\
\mathbf{n}(\%)\end{array}$ & $\begin{array}{c}\text { Feminino }(\mathbf{n}=\mathbf{9 7}) \\
\mathbf{n}(\%)\end{array}$ \\
\hline Sem dor (0) & $3(1,7)$ & $3(3,9)$ & - \\
Dor leve (1 a 3) & $36(20,7)$ & $16(20,8)$ & $27(20,6)$ \\
Moderada (4 a 6) & $50(28,7)$ & $32(29,8)$ \\
Forte (7 a 9) & $77(44,3)$ & $3(3,9)$ & $45(46,4)$ \\
Insuportável (10) & $8(4,6)$ & $5(5,2)$ \\
\hline
\end{tabular}

A tabela 2 destaca as medianas e o intervalo interquartil das variáveis de dor lombar autorreferida pelos trabalhadores rurais nos testes Oswestry e EVA, bem como flexibilidade toracolombar e de isquiotibiais, de acordo com a classificação do NAF - Ipaq.

Tabela 2. Dor lombar, flexibilidade toracolombar e de isquiotibiais em trabalhadores rurais de acordo com o nível de atividade física

\begin{tabular}{lrrrrr}
\hline Variável & \multicolumn{1}{c}{$\begin{array}{c}\text { Total } \\
\mathbf{n}=\mathbf{1 7 4}\end{array}$} & \multicolumn{1}{c}{$\begin{array}{c}\text { AMA } \\
\mathbf{n}=\mathbf{6 2}\end{array}$} & \multicolumn{1}{c}{$\begin{array}{c}\text { IA } \\
\mathbf{n}=\mathbf{5 4}\end{array}$} & \multicolumn{1}{c}{$\begin{array}{c}\text { SED } \\
\mathbf{n}=\mathbf{5 8}\end{array}$} & $\mathbf{p}$ \\
\hline Idade & $47(42-52,5)$ & $48(42-53)$ & $46(40-52)$ & $45(42-53)$ & 0,438 \\
Oswestry & $5(1-10)$ & $4,5(2-9)$ & $6(2-9,25)$ & $4(0-11)$ & 0,590 \\
EVA & $6(4-8)$ & $6,5(5-8)$ & $6,5(4-8)$ & $6(4-7)$ & 0,441 \\
AP & $120(110-130)$ & $125(115-135)$ & $120(115-130)$ & $120(110-125)$ & $\mathbf{0 , 0 0 5 ^ { \star }}$ \\
TEPR & $65(60-70)$ & $70(60-76,25)$ & $65(60-70)$ & $60(60-70)$ & 0,144 \\
FBW & $22(20-25)$ & $22,3(20-25)$ & $22,65(20-25)$ & $22(20,5-23)$ & 0,434 \\
\hline
\end{tabular}

AMA: Ativos ou Muito Ativos. IA: Insuficientemente Ativos. SED: Sedentários. EVA: Escala Visual Analógica da dor. AP: Ângulo Poplíteo. TEPR: Teste de Elevação da Perna Retificada. FBW: Flexibilidade pelo Banco de Wells. p: Estatística de Kruskall Wallis.

* Nível de Significância: $p<0,05$.

Quando comparados os dados multiplamente (U de Mann-Whitney) entre os grupos, verificou-se diferença estatisticamente significativa entre os indivíduos sedentários e insuficientemente ativos $(\mathrm{p}=0,032)$ e sedentários e ativos ou muito ativos $(p=0,002)$. Dessa forma, indivíduos sedentários possuem menores valores de flexibilidade de isquiotibiais do que indivíduos que realizam atividades físicas.

Não houve diferenças significativas nas demais comparações de flexibilidade e ou dor entre os grupos.

A tabela 3 apresenta os dados comparativos das variáveis entre trabalhadores homens e mulheres. 
Tabela 3. Dor lombar, flexibilidade toracolombar e de isquiotibiais em trabalhadores rurais homens e mulheres de acordo com o nível de atividade física

\begin{tabular}{|c|c|c|c|c|c|c|}
\hline & Variável & $\begin{array}{l}\text { Total } \\
n=77\end{array}$ & $\begin{array}{l}\text { AMA } \\
n=20\end{array}$ & $\begin{array}{c}\text { IA } \\
n=18\end{array}$ & $\begin{array}{c}\text { SED } \\
\mathrm{n}=39\end{array}$ & $\mathrm{p}$ \\
\hline \multirow{7}{*}{ 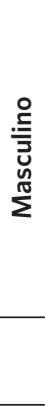 } & Idade & $48(42-52,75)$ & $48(47-55,5)$ & $48(39-51)$ & $45(42-52)$ & 0,160 \\
\hline & Oswestry & $4(0-9)$ & $5(2-8)$ & $5,5(2-9)$ & $4(0-10)$ & 0,596 \\
\hline & EVA & $6(3,5-7)$ & $6(3,5-7,5)$ & $5(4-8)$ & $6(3-7)$ & 0,982 \\
\hline & AP & $120(115-130)$ & $125(120-135)$ & $126,5(120-130)$ & $120(110-125)$ & $0,007^{\star}$ \\
\hline & TEPR & $65(60-72,5)$ & $70(60-70)$ & $65(60-80)$ & $65(60-75)$ & 0,659 \\
\hline & FBW & $22(20,25-24,25)$ & $22,65(21-25,5)$ & $22(20-23)$ & $21,75(20,5-24,5)$ & 0,625 \\
\hline & Variável & $\begin{array}{l}\text { Total } \\
\mathrm{n}=97\end{array}$ & $\begin{array}{l}\text { AMA } \\
n=42\end{array}$ & $\begin{array}{c}\text { IA } \\
n=36\end{array}$ & $\begin{array}{l}\text { SED } \\
n=19\end{array}$ & $\mathrm{p}$ \\
\hline \multirow{6}{*}{. } & Idade & $46(40,5-52,5)$ & $46,5(41-52)$ & $46(40-52)$ & $46(42-53)$ & 0,943 \\
\hline & Oswestry & $6(2-11)$ & $4,5(1-10)$ & $6,5(2-12)$ & $6(2,5-15)$ & 0,754 \\
\hline & EVA & $7(4-8)$ & $7(5-8)$ & $7(4,5-8)$ & $6(4-7)$ & 0,427 \\
\hline & AP & $120(110-130)$ & $125(110-130)$ & $120(110-127,5)$ & $120(110-122,5)$ & 0,062 \\
\hline & TEPR & $65(60-70)$ & $70(60-80)$ & $67,5(60-70)$ & $60(57,5-60)$ & 0,071 \\
\hline & FBW & $22,3(20-25)$ & $22,15(19,3-25)$ & $23,5(20-25,5)$ & $22(20,5-23)$ & 0,288 \\
\hline
\end{tabular}

AMA: Ativos ou Muito Ativos. IA: Insuficientemente Ativos. SED: Sedentários. EVA: Escala Visual Analógica da dor. AP: Ângulo Poplíteo. TEPR: Teste de Elevação da Perna Retificada. FBW: Flexibilidade pelo Banco de Wells. p: Estatística de Kruskall Wallis.

* Nível de Significância: $p<0,05$

Na comparação dos dados entre os grupos (U de Mann-Whitney), verificou-se diferença estatisticamente significativa entre os homens sedentários e insuficientemente ativos $(p=0,007)$ e sedentários e ativos ou muito ativos ( $\mathrm{p}=0,013)$, sendo que os sedentários possuem menores valores de flexibilidade de isquiotibiais dos trabalhadores que realizam atividades físicas. Mulheres sedentárias possuem menores valores de flexibilidade de isquiotibiais do que as ativas ou muito ativas tanto no teste de AP $(\mathrm{p}=0,024)$ quanto no TEPR $(\mathrm{p}=0,039)$.

Não houve diferenças significativas nas demais comparações de flexibilidade e/ou dor entre os grupos.

Observou-se correlação negativa nas medidas de flexibilidade toracolombar e de isquiotibiais pelo AP, TEPR e FBW com a dor pela EVA, conforme figura 1 . 
Figura 1. Correlação entre a flexibilidade toracolombar e de isquiotibiais com o grau de dor pela EVA
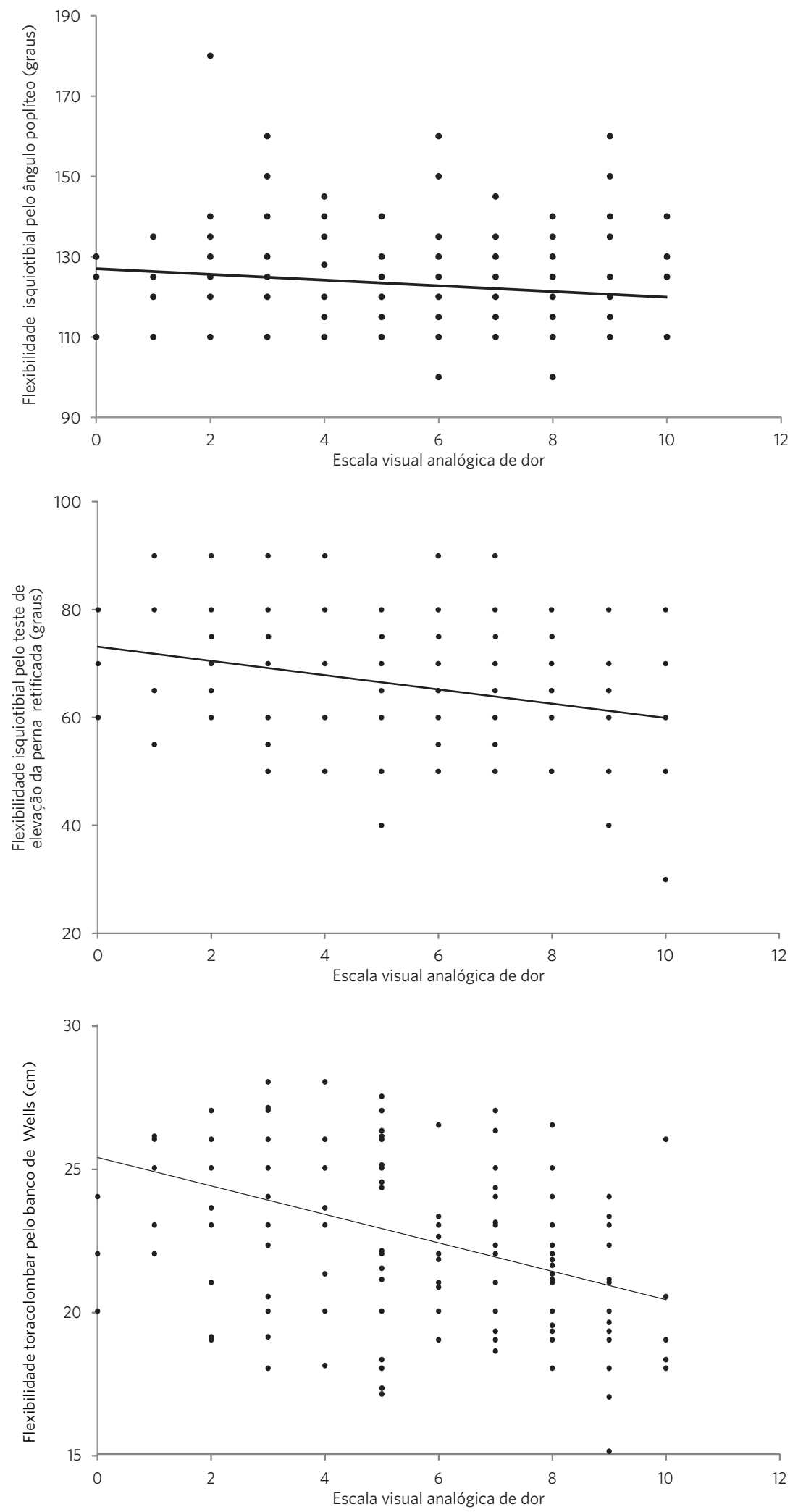

$r(s)=-0,42 ; p=0,0001$ 
Não se observou correlações estatisticamente significativas entre a escala da dor (EVA) com o número de horas relatadas pelos trabalhadores durante o período da safra $(\mathrm{r}=-0,06 ; \mathrm{p}=0,956)$ e fora do período de safra $(r=0,08 ; p=0,442)$.

\section{Discussão}

A prevalência de dor lombar foi alta entre os agricultores pesquisados (98,3\%), e nas mulheres foi de $100 \%$. A média de dor pela EVA foi de $5,89( \pm 2,49)$ e mais alta nas mulheres $(6,14 \pm 2,45)$, em relação aos homens $(5,56 \pm$ 2,51). Carvalho e Kovacs (2006) classificam o sintoma de dor como moderada, ou seja, pode interferir parcial ou totalmente no desempenho das atividades.

Os resultados são superiores aos de O'Sullivan, Cunningham e Blake (2009) que identificaram $74 \%$ de prevalência de dor lombar em 104 agricultores irlandeses; Birabi, Dienye e Ndukwu (2012), com $67,10 \%$, em um estudo com 310 agricultores saudáveis no sul da Nigéria.

Dos trabalhadores que experimentaram dor lombar, $72 \%$ afirmaram que a agricultura contribuiu com seu problema e interferiu na realização do trabalho diário dos indivíduos. Atividades que incluem o levantamento de objetos pesados foi o fator limitante mais citado, seguido da condução de máquinas, do trabalho com gado e dosagem dos animais (O'SULLIVAN; CUNNINGHAM; BLAKE, 2009).

$\mathrm{O}$ presente estudo não encontrou correlação entre a intensidade da dor lombar e número de horas em atividades agrícolas, no entanto Birabi, Dienye e Ndukwu (2012) relatam que a dor lombar é mais prevalente naqueles que exercem atividade agrícola de longa duração. Os autores complementam ainda que existe alta prevalência de dor lombar na faixa etária de 31-40 anos (49,04\%) e no sexo feminino (50,96\%).

Sabe-se que entre as enfermidades crônicas que acometem as mulheres, uma das mais comuns é a dor lombar, principalmente no período pós-menopausa, já que nessa fase há alteração na composição corporal, com maior presença de gordura e menor massa magra, o que pode contribuir para a intensidade dos quadros álgicos (MARASCHIN ET AL., 2010). $\mathrm{O}$ avanço da idade, associado a uma atividade que exige esforço físico, de forma contínua, pode predispor essas mulheres a maior prevalência de dor, como no caso deste estudo. $\mathrm{O}$ envelhecimento é uma tendência que envolve a população rural no estado de Santa Catarina dada a migração dos jovens para as cidades. Essa realidade pode explicar os valores mais elevados da escala de dor em trabalhadores rurais em relação a outros estudos.

A classificação de Oswestry indicou disfunção leve na maioria dos trabalhadores (78,2\%), no entanto pela classificação da EVA há predomínio de dor forte (entre sete e nove na EVA) tanto na população em geral $(44,3 \%)$ quanto em homens (41,6\%) e mulheres (46,4\%), o que impediria o desempenho das atividades (CARVALHO; KOVACS, 2006). Esse fator pode ser considerado como contraditório entre os instrumentos de medidas para verificação da dor lombar, ou demonstrar a necessidade de execução das tarefas laborais, mesmo com dores elevadas.

Estudo de Liu et al. (2012) investigou a prevalência de dor nas costas autorrelatadas e as associações entre potenciais fatores de risco e dor nas costas com 2.045 agricultores chineses selecionados a partir de 800 famílias. As associações entre dor nas costas e potenciais fatores de risco foram realizadas por regressão logística e incluíram idade, sexo, nível de educação, estresse percebido, principais atividades agrícolas, tabagismo e hábito de beber. Da população estudada, 786 (38,4\%) agricultores relataram dor nas costas. Dois terços das pessoas com dor nas costas $(66,0 \%)$ relataram que a dor afeta a quantidade e qualidade do trabalho. $\mathrm{O}$ relato de dor aumenta com a idade, e mulheres e agricultores que sofreram algum estresse regular eram mais propensos a relatar dor 
nas costas. Ainda, a dor nas costas autorrelatada foi mais frequentemente entre os fumantes e os que consumiam bebidas alcoólicas.

Pesquisa de Silva et al. (2013) observou que há déficit de conhecimento dos agricultores quanto à exposição aos riscos em função do seu trabalho, fator que preocupa, visto que, para assumir uma postura de cuidado, o sujeito precisa reconhecer os aspectos que o colocam em condição vulnerável perante as questões ocupacionais. No caso de dor lombar, programas como escola postural e grupos de algia para agricultores podem se constituir em estratégias eficazes para diminuir os índices de dor lombar, mas, inicialmente há que se mobilizar esforços para sensibilizar os trabalhadores quanto aos riscos a fim de promover adesão destes aos grupos de apoio e tratamento.

Soysal, Kara e Arda (2013), ao investigar o NAF pelo Ipaq em pacientes com lombalgia crônica e dor cervical em 96 pacientes, divididos igualitariamente em três grupos - pacientes com dor lombar ou cervical no pré-operatório, ambulatoriais e controles saudáveis -, observaram que houve modificação de atividade física em pacientes com lombalgia crônica e dor cervical e que os pacientes pré-operatórios com lombalgia são mais afetados do que aqueles com problemas cervicais.

Manter-se ativo, com bons níveis de funcionalidade, são fatores que protegem a saúde do ser humano, desde que as atividades físicas não gerem sobrecarga e que sejam realizadas com orientações para evitar a sobrecarga e posicionamento inadequado.

Pesquisa de Johnson e Thomas (2010) relata que indivíduos com lombalgia ou história de dor lombar crônica não apresentaram correlação significativa entre a flexibilidade dos isquiotibiais medida pelo TEPR e a excursão de flexão lombar em tarefas de flexão de tronco, sendo razoável supor que o aumento do comprimento dos isquiotibiais não afetaria a mobilidade lombar em tarefas que exijam flexão de tronco em indivíduos com lombalgia. No entanto, o presente estudo observou correlações significativas entre a flexibilidade dessa musculatura e a intensidade de dor lombar.

Halbertsma et al. (2001) investigaram a extensibilidade e rigidez dos isquiotibiais em pacientes com dor lombar não específica em 40 indivíduos, sendo 20 pacientes com dor lombar não específica e 20 indivíduos saudáveis. $\mathrm{O}$ grupo de pacientes com dor lombar apresentou restrição significativa na ADM e extensibilidade dos isquiotibiais em comparação com o grupo controle e não houve diferença significativa na rigidez muscular dos isquiotibiais entre os grupos. Dessa forma, a diminuição da ADM e extensibilidade dessa musculatura não está relacionada com a rigidez, mas sim com a tolerância ao alongamento dos pacientes.

O estudo encontrou correlações negativas significativas na flexibilidade toracolombar e de isquiotibiais em todas as medidas analisadas. Destaca-se a correlação observada na avaliação da flexibilidade toracolombar, medida pelo Banco de Wells no teste de sentar e alcançar, que foi considerada moderada $(r=-0,422, p<0,001)$, sendo que as medidas do AP e TEPR foram consideradas fracas $(r=-0,153, p=0,044 ; r=-0,238$, $\mathrm{p}=0,002$ ).

Pacientes com dor lombar possuem menor ADM, alterações na rigidez muscular e déficit nos níveis de razão de força concêntrico/ excêntrica. A diminuição da extensibilidade dos isquiotibiais foi associada ao aumento da rigidez passiva durante a $\mathrm{ADM}$ comum de movimento $\left(20^{\circ}\right.$ a $\left.50^{\circ}\right)$, e a tolerância ao alongamento está associada à restrição mecânica, e não a medidas comportamentais autorreferidas que indicam aumento da dor ou medo. Não há clareza no estudo se a diminuição da extensibilidade dos isquiotibiais é causa ou efeito na dor lombar crônica (MARSHAL; MANNION; MURPHY, 2009).

Os quadros de dor lombar, sejam eles crônicos ou agudos, geram sofrimento e limitações ao trabalhador rural. Conforme estudo de Medina et al. (2014), são poucas as ações de 
promoção da saúde e prevenção de doenças crônicas organizadas pelas equipes de atenção primária no País, principalmente no meio rural, o que, por si só, é suficiente para colocar como premente a adoção de medidas que minimizem essa realidade. A Fisioterapia pode contribuir atuando no tratamento das algias, com programas de reabilitação ou de educação em saúde e prevenção, com vistas a minimizar o sofrimento gerado por essa condição. A população rural e trabalhadora, que apresenta um quadro tão prevalente de dor, carece de orientações e assistência de uma equipe multiprofissional para minimizar a realidade encontrada neste estudo.

Para que as ações sejam efetivas, há que se considerar os determinantes sociais do contexto rural e a concepção ampliada de saúde, bem como reconhecer o protagonismo dos sujeitos na construção da sua saúde. Por outro lado, é preciso reforçar a responsabilidade do Estado na produção de condições de vida condizentes com as necessidades da população, para não afirmar uma tendência de culpabilizar e responsabilizar o indivíduo pela sua saúde (SILVA; BAPTISTA, 2015). Residir em meio rural, em algumas regiões, restringe o acesso dos sujeitos aos programas de saúde, o que, de fato, interfere nas condições de vida dessa população, aspecto que precisa ser modificado mediante políticas que atentem para as especificidades desse segmento.

\section{Conclusões}

Dos agricultores pesquisados, 98,3\% relataram algum sintoma de dor lombar, sendo presente em $100 \%$ das mulheres.

Houve relação estatisticamente significativa entre a flexibilidade de isquiotibiais medida pelo AP e o NAF dos trabalhadores, indicando que indivíduos sedentários possuem grau significativamente menor de AP do que os insuficientemente ativos e ativos ou muito ativos. O mesmo resultado ocorre entre os homens; e as mulheres sedentárias possuem menor flexibilidade nos testes de AP e TEPR do que as ativas e muito ativas.

Observou-se correlação negativa moderada entre a intensidade da dor e a flexibilidade toracolombar pelo FBW e fraca de isquiotibiais pelo AP e TEPR.

Resultados contraditórios de associação entre a dor lombar e flexibilidade demonstram que novos estudos explorem essas variáveis, bem como a verificação da $\mathrm{ADM}$ da coluna vertebral com atividades realizadas em trabalhadores e a adoção de programas de exercícios educativos e preventivos com trabalhadores rurais.

Dada a quantidade e a diversidade de atividades físicas realizadas pelos agricultores, os resultados deste estudo também indicam a necessidade de estudos adicionais de avaliação da atuação das equipes multiprofissionais de saúde da Estratégia Saúde da Família e do Núcleo de Apoio à Saúde da Família, tendo como foco esta população.

\section{Colaboradores}

Marcia Regina da Silva contribuiu substancialmente em todas as etapas do manuscrito; Fátima Ferretti contribuiu com a Concepção e interpretação dos dados, produção e revisão crítica do artigo e seu conteúdo intelectual, aprovação final da versão a ser publicada; Junir Antonio Lutinski contribuiu com a análise e interpretação dos dados; revisão crítica do conteúdo intelectual e aprovação final da versão a ser publicada. 


\section{Referências}

AFFONSO FILHO, A. A.; NAVARRO, R. D. Avaliação do ângulo poplíteo em joelhos de adolescentes assintomáticos. Rev Bras Ortop, São Paulo, v. 37, n. 10, p. 461-466, out. 2002.

ALVES, R. A.; GUIMARÃES, M. C. De que sofrem os trabalhadores rurais? Análise dos principais motivos de acidentes e adoecimentos nas atividades rurais. Informe Gepec, Toledo, v. 16, n. 2, p. 39-56, jul./dez. 2012.

BERTOLLA, F. et al. Efeito de um programa de treinamento utilizando o método Pilates ${ }^{\circledR}$ na flexibilidade de atletas juvenis de futsal. Rev Bras Med Esporte, Niterói, v. 13, n. 4, p. 222-226, ago. 2007.

BIRABI, B. N.; DIENYE, P. O.; NDUKWU, G. U. Prevalence of low back pain among peasant farmers in a rural community in South South Nigeria. Rural Remote Health, Geelong, v. 12, n. 1, p. 1912-1920, ago. 2012.

CARVALHO, D. S.; KOWACS, P. A. Avaliação da intensidade de dor. Migrâneas cefaleias, Curitiba, v. 9, n. 4, p. 164-168, out./dez, 2006.

FATHALLAH, F. A. Musculoskeletal disorders in labor-intensive agriculture. Appl Ergon, London, v. 46, n. 6, p. 738-743, out. 2010.

FERREIRA, G. D. et al. Prevalência de dor nas costas e fatores associados em adultos do sul do Brasil: estudo de base populacional. Rev. Bras. Fisioter, São Paulo, v. 15, n. 1, p. 31-6, fev. 2011.

GAYA, A.; SILVA, G. Projeto esporte Brasil: manual de aplicação de medidas e testes, normas e critérios de avaliação. Porto Alegre: Proesp-br, 2009.

HALBERTSMA, J. P. et al. Extensibility and stiffness of the hamstrings in patients with nonspecific low back pain. Archives of physical medicine and rehabilitation, Philadelphia, v. 82, n. 2, p. 232-238, 2001.

HAMILL, J.; KNUTZEN, K. M. Bases biomecânicas do movimento humano. 3 ed. São Paulo: Manole, 2012.

\section{INSTITUTO BRASILEIRO DE GEOGRAFIA E} ESTATÍSTICA (IBGE). Cidades. Disponível em: $<$ http://cidades.ibge.gov.br/xtras/perfil.php?lang=\&c odmun $=420410 \&$ search $=$ santa- catarina $\mid$ caxambu-do-sul>. Acesso em: 18 jul. 2016.

JOHNSON, E. N.; THOMAS, J. S. Effect of hamstring flexibility on hip and lumbar spine joint excursions during forward-reaching tasks in participants with and without low back pain. Archives of physical medicine and rehabilitation, Philadelphia, v. 91, n. 7, p. 1140-1142, 2010 .

KENDALL, F. P.; MCCREARY, E. K.; PROVANCE, P. G. Músculos, provas e funções: com postura e dor. 5. ed. São Paulo: Manole, 2007. 556 p.

LIU, X. et al. Back pain among farmers in a northern area of China, Spine, Lebanon, v. 37, n. 6, p. 508-514, mar. 2012

MARASCHIN, R. et al. Dor lombar crônica e dor nos membros inferiores em idosas: etiologia em revisão. Fisioter. mov, Curitiba, v. 23, n. 4, p. 627-639, out./dez. 2010.

MARSHALL, P. W. M.; MANNION, J.; MURPHY, B. A. Extensibility of the hamstrings is best explained by mechanical components of muscle contraction, not behavioral measures in individuals with chronic low back pain. PMER, New York, v. 1, n. 8, p. 709-718, 2009.

MASSELI, M. R.; LOPES, M. M.; SERILLO, T. B. Índice funcional de Oswestry de pacientes submetidos à cirurgia para descompressão de raízes nervosas. Rev. Fisioter. Univ. São Paulo, São Paulo, v. 10, n. 2, p. 70-76, jul./dez. 2003.

MATSUDO, S. M. et al. Questionário internacional de atividade física (IPAQ): estudo de validade e reprodutibilidade no Brasil. Revista Brasileira de Atividade Física Et Saúde, Pelotas, v. 6, n. 2, p. 5-18, 2001. 
MATSUDO, S. M.; MATSUDO, V. K. R.; BARROS

NETO, T. L. Atividade física e envelhecimento: aspec-

tos epidemiológicos, Rev Bras Med Esporte, Niterói, v. 7, n. 1, p. 2-13, 2001.

MEDINA, M. G. et al. Promoção da saúde e prevenção de doenças crônicas: o que fazem as equipes de Saúde da Família? Saúde debate, Rio de Janeiro, v. 38, n. esp., p. $69-82$, out. 2014 .

MOREIRA, J. P. L. et al. A saúde dos trabalhadores da atividade rural no Brasil. Cad. Saúde Pública, Rio de Janeiro, v. 31, n. 8, p. 1698-1708, ago. 2015.

O'SULLIVAN, D.; CUNNINGHAM, C.; BLAKE, C. Low back pain among Irish farmers. Occupational medicine, London, v. 59, n. 1, p. 59-61, ago. 2009.

SILVA, J. B. et al. Fumicultores da zona rural de Pelotas (RS), no Brasil: exposição ocupacional e a utilização de equipamentos de proteção individual (EPI). Saúde debate, Rio de Janeiro, v. 37, n. 97, p. 347-353, jun. 2013.
SILVA, P. F. A.; BAPTISTA, T. W. F. A Política Nacional de Promoção da Saúde: texto e contexto de uma política. Saúde debate, Rio de Janeiro, v. 39, n. esp, p. 91-104, dez. 2015.

SOYSAL, M.; KARA B.; ARDA M. N. Assessment of physical activity in patients with chronic low back or neck pain. Turkish neurosurgery, Ankara, v. 23, n. 1, p. $75-80,2013$.

VIDMAR, M. F. et al. Atividade física e qualidade de vida em idosos. Revista saúde e Pesquisa, Maringá, v. 4, n. 3, p. 417-424, set./dez. 2011.

ZANCHETTA, L. M. et al. Inatividade física e fatores associados em adultos, São Paulo, Brasil. Revista Brasileira de Epidemiologia, São Paulo, v. 13, n. 3, p. 387-399, set. 2010.

Recebido para publicação em agosto de 2016

Versão final em janeiro de 2017

Conflito de interesses: inexistente

Suporte financeiro: Bolsa de auxílio à pesquisa pela modalidade art. 170 da Constituição Estadual para Núcleos de Iniciação

Científica 